\section{Social Engagement versus Isolation in the Well-being of Individuals with Autism Spectrum Disorder}

\author{
Keesha K Jones* \\ College of Psychology, Nova South-Eastern \\ University, Fort Lauderdale, Florida, USA
}

*Corresponding author: Keesha K. Jones

” Kj588@mynsu.nova.edu

College of Psychology, Nova Southeastern University, Fort Lauderdale, Florida, USA.

Tel: $800-541-6682$

Citation: Jones KK (2019) Social

Engagement versus Isolation in the Wellbeing of Individuals with Autism Spectrum Disorder. J Child Dev Disord. Vol.5 No.3:9

\section{Introduction}

Autism Spectrum Disorder (ASD) is a pervasive neurodevelopmental disorder characterized by impairments in social communication and restricted, repetitive patterns of behavior, interests or activities (American Psychiatric Association, 2013). ASD depicts a group of varied development disorders that comprise diverse symptoms, skill sets, as well as disability levels (National Institutes of Mental Health, 2018). Autism Individuals who have been diagnosed with this disorder exhibit the following characteristics: constant social challenges, especially in communication; recurrent ambivalent behaviors coupled with a low interest in activities, as well as disorganized social functioning that mainly affect daily activities such as school or work. The ability to adapt to this condition and the well-being of such individuals is affected by several factors. Social integration is one of the key factors that affect their well-being. On the contrary, social isolation also impacts the well- being of these individuals in a separate way.

In this paper, a brief evaluation of how social integration versus social isolation impacts the well- being of individuals with this ASD will be examined. For the purpose of this review, social integration is defined as the extent to which an individual participates in a broad range of social roles and relationships [1]. Similarly, social engagement refers to an individual's participation in activities of a social group [2]. Researchers studied how social engagement leads to a better quality of life and functioning in individuals on the spectrum [3]. Whereas adults with ASD who were socially isolated, meaning there was a lack of opportunities to be social and maintain social relationships, were found to have poor outcomes such as loneliness and decreased social functioning. Social isolation is highest among individuals with ASD compared to other disability groups; however, those who live independently are more likely to have social relationships and have more opportunities for social relationships [3] Personal and contextual factors in limited social engagement were identified in a large diverse nationally representative study of young adults with autism [3]. Identified factors were communication and functional impairment, and living with family rather than independently; 
those who live independently were more likely to get together with friends, though it is not clear why [3].

\section{Literature Review}

\section{Benefits and difficulties for individuals with ASD with social integration}

The ability to develop social skills through group mediated activities such as skills training groups, vocational training, sports, and community engagements (i.e., cultural/religious events), brings a sense of belonging to an individual diagnosed with ASD. This culminates in the establishment of close relationships with their peers and allows for the individual to become integrated into a peer social group. As a result, some of the symptoms of this disorder may start to dissolve as individuals can more easily communicate and develop an interest in peer-group activities. As such, social integration within a peer group enhances their ability to engage socially, thereby translating to the overall well-being of these individuals. Conversely, when the individuals become isolated and are unable to interact effectively with friends through such group interaction, their overall well-being is thwarted. Social integration fosters the development of social understanding and knowledge on how to relate to each other [4]. Notably, it fosters the enhancement of social functioning whereby the individuals with ASD become well adapted to function effectively in a social group. Also, social engagement has the ability to decrease the level of loneliness as well as any likely psychiatric symptoms due to alienation. Researchers examined ten studies in which group social skills interventions led to enhanced social understanding, improved social functioning, reduced loneliness, and alleviated co-morbid psychiatric symptoms [4].

Taking an active part in groups that offer training for social skills is a vital aspect that is critical for adults diagnosed with ASD. This may involve interventions led by multiple therapists that offer training to a small group of individuals diagnosed with ASD. As such, these individuals are taught certain social skills and are then required to rehearse as well as reinforce them [5]. Through this training, they become fully integrated into these social groups and develop cognitive social skills that make them able to associate with others. The resultant effect is the improvement of the overall well-being of individuals with ASD. On the contrary, the inability to accommodate the ASD individuals into groups that train social skills would result in thwarting the overall well-being of these individuals. A study on the PEERS caregiver implemented social intervention suggested that good social skills and social support correlate with a better quality of life, and poor relationships contribute to loneliness and other mental health problems [6].

\section{Problems with isolation}

National Autistic Society, (2017) identifies that adults with ASD are likely to undergo social isolation related to a couple of reasons. These may include personal preference of remaining alone, lack of social skills to engage with others, inability to sustain appropriate social behaviors, previous bad experiences, lack of vital support, as well as poor social networks. The overall effect is the impairment of the overall well-being of such individuals, thereby fostering a negative outcome and an inability to cope with social challenges. Poor social interaction results in social isolation in most areas, and as such individuals are unable to relate effectively to others. Similarly, social isolation prevents individuals on the spectrum from becoming integrated into social groups as well as groups with special interests.

For instance, it becomes incredibly difficult for them to engage in activities such as sports, religious events, or any vocational based activities. As a result, these individuals become socially isolated and as such, they are likely to develop loneliness that may translate to comorbid mental health problems such as depression. This mechanism is explained to be the result of characteristics of ASD such as fear of change, rituals, need for sameness that may interfere with the ability to interact socially and a possible bidirectional influence of impaired ability to interact socially causing anxiety or anxiety due to difficulty with social interaction [7]. Depression is explained to often be masked by core characteristics of ASD such as flat affect, self-injurious behavior and social withdrawal, in which depression is conceived to be expressed in adolescence/early adulthood as a result of an individual's increased awareness of their social difficulties and challenges $[8,9]$. Likewise, their intellectual, emotional, psychological, as well as physical well-being is significantly affected.

Lack of support to become fully integrated into social activities is another notable challenge that faces the ASD population translating into isolation. Such support may be obtained from well- organized community social groups, workgroups or event family involvement. Often, the family forms the basic unit of interaction in an individual. In situations where the family is minimally involved in conscious activities to foster the well-being of such individuals, they become isolated and their overall wellbeing is greatly affected. Some of the support programs may entail the involvement in befriending schemes where volunteers may spend significant time with the ASD adults in offering training on how to enhance social skills.

Furthermore, isolation from the family circle is likely to result in hopelessness that would in turn foster loss of connection with other social groups. It is due to this loneliness and isolation that negative social outcomes such as social conflicts are likely to sprout. Moreover, the lack of well-structured community-based programs that aim to aid individuals with ASD is likely to raise the level of isolation among these individuals. Eventually, the overall well-being of such individuals may become greatly compromised especially when they become socially isolated. It is worth noting that the lack of effective social networks in the community as well as in the places of work is likely to translate to social isolation among the adults with ASD [10]. This is because this robs these individuals the opportunity to meet friends and groups of people who are likely to offer individualized support to meet personal objectives in life. The resulting effect is profound alienation and dissatisfied social relevance among such individuals. Eventually, negative symptoms of isolation develop such as depression and inappropriate social behaviors. Therefore, isolation deteriorates 
the well-being of individuals with ASD, hence translating to poor socialization.

\section{Strategies for Social Integration}

\section{Social skills interventions}

Social skills interventions such as PEERS can help to improve the quality of life for families of individuals with ASD and as a result, increase the well-being of these individuals. Also, the PEERS intervention adds to the well-being of adolescents with ASD through its use of cognitive-behavioral techniques in improving social functioning. Through PEERS social skills intervention, families are involved in the development of their family member's social skills and thus are relieved of some stress and emotional strain because they are adapting positively in being a part of the social learning process.

A study consisting of 64 parent-child dyads, 32 in the PEERS intervention and 32 in a control group, examined family chaos, parental stress, and parental self-efficacy in the 32 parentchild in both group dyads after 14 weekly sessions of the PEERS curriculum. PEERS curriculum required that adolescents schedule at least two extracurricular activities, have a weekly get-together with other teens and their parents and call friends from school or community groups. It was found that parents who participated in the PEERS intervention compared to the control, showed decreased parental stress, as predicted. A study of the Korean version of the PEERS intervention highlighted that the social integration among individuals with ASD can be enhanced through programs for educating and enriching the ASD adults with relational skills [11]. These programs mainly aim at improving the ability of individuals to make new friends through the development of effective social relation skills. This ability to more easily and effectively develop new friends fosters effective social integration and thereby nurtures a sense of belonging. Such programs equip ASD individuals with sound skills on how to handle rejection as they build-up their social integration skills [11]. The ability to develop sound skills in handling social rejection is vital in enhancing the well-being of adults with ASD.

Additionally, these social skills intervention programs such as PEERS, equip individuals on the spectrum with conflict resolution skills pertinent to enhancing their well-being. Often, since individuals diagnosed with ASD can lack effective social skills, they are likely to encounter conflicts likely to result in either physical or emotional harm. Therefore, in undergoing social skills intervention programs individuals with ASD are taught about how to prevent conflicts from arising and how to handle such conflicts. Eventually, the well-being of these individuals is improved since they are well trained in skills to prevent and handle any possible conflict that would come about following such interactions. In contrast, failure to develop new friendships, effectively manage conflicts, as well as poor ability in handling rejection is likely to result in social isolation and thereby translate to impaired wellbeing.

Research suggests that families of individuals with ASD can take on maladaptive coping mechanisms associated with factors that accompany supporting an individual with ASD such as time pressures, financial burdens, additional attention, that can create stress and disorganization in families raising a child with ASD [12]. The presence of maladaptive coping can create conflict within families that exacerbates behavioral problems in individuals with ASD. With behavioral problems made worse, there is an added barrier to participation in activities, as limited engagement in community activities has been seen in families with this conflict [13]. Also, increased behavioral problems take away from the individual's ability to learn social skills and result in reduced opportunities for social engagement.

Another social skills intervention to increase social interactions for students with autism and their peers without ASD in an integrated first-grade classroom has been studied [14]. Within this study, there were social skills groups in which participants were trained in initiating, responding, keeping interactions going, greeting others, giving and accepting compliments, taking turns and sharing, asking for help and helping others, and including others in their conversation. The study was a multiple baseline across subject's design, and training took place four times weekly. The social skills intervention resulted in increased frequency of, time engaged in, and duration of social interactions, as well as increased social engagement between participants and their peers.

Furthermore, their research is supporting the use of interventions for increasing social interaction through cooperative learning and social skills groups with embedded peer training [14]. Peer training for the social skills group targeted initiation of conversations and responses to peers, working together with peers, and positively interacting with peers in play activities [14]. Increased social interaction was seen between children with ASD and their peers without ASD, and post-intervention generalization of the learned skills was observed. The frequency and length of time of social interaction increased during both socials skills and cooperative learning groups. It was found in their next study that cooperative learning and social skills group with peers had shown better generalization to other settings in thirty-four students with autism [14].

Researchers examined a peer-mediated intervention delivered during school lunch that resulted in improvements in the conversational skills of high school students with ASD [15]. All participants had improved conversational abilities with their peers, more often engaging in conversational acts and having longer conversations post-intervention. Participants also showed improved ability to initiate conversations and keep conversations going through the use of follow-up questioning. Also, the intervention leads to increased use of comments, a skill taught to keep conversations going and became more active in the conversations they had rather than passive or reluctant [15]. Moreover, educator asserted the conversations that had taken place to be natural and typical of high school conversations, confirming that the interactions between participants and their peers were of value [15].

The outcomes of this study are significant in that participants who were once socially isolated, rarely engaging in conversation with 
peers, were seen to hold and engage in conversations in a natural way. Researchers credited the increase in the use of follow-up questions, a skill taught to keep conversations going, to the use of cue cards [15]. In this study, the primary interventionists were the individuals' peers, all trained outside of the lunch activity to implement intervention without supervision. It was noted that through training their peers, they can better support social conversations with students with ASD in other activities during the day [15]. The peers were trained in five 30-min group training sessions and took part in $5 \mathrm{~min}$ of daily feedback to improve conversations. The cue cards used in this intervention had scripted text to prompt student initiations around ageappropriate topics of conversations that helped participants to initiate conversations and possibly reduce the need for prompts.

\section{Mobile Apps}

In a world where technological advances are continuously being made, it is important to consider how technology could be used in promoting social engagement in individuals with ASD. A study focused on the HANDS app to examine how mobile technology can support social functioning and increase social engagement in a group of 26 children [16]. Initially, there were a series of sessions focused on familiarizing students with technology. The HANDS app utilizes flexible web-based software that teachers can use to improve on particular areas of support and individualized intervention arrangements for each child [16]. The arrangement of sequences on the app is linked to screens in which the text, images, video, and sounds are customizable, and there is also a diary associated with each sequence for use. An example of a sequence is "getting to the bus independently", in which there are images with associated step by step text and sound prompts. Another notable feature of the HANDS app is the recording of each time the app is used by the child.

Findings from the study found that at least 15 of the participants preferred the use of the HANDS app for prompts, rather than prompts from their teacher. The study suggested that this preference may be due to cognitive impairments in processing speeds that are seen in children with autism [16]. However, this reasoning was only implicitly or explicitly supported by teachers or students in 3 cases. In one case, a child using HANDS to support his development of the life skills task of making toast independently was observed to successfully complete this task whilst using HANDS as support. The child was noted to prefer hearing instruction from the voices provided by the app rather than their teacher. It was suggested by this child's teacher that this is because he "can do it in his own time" and is able to take as long as he needs to process the individual intervention messages from the mobile device and can control when to move on to further instructions of the intervention [16]. Other findings from the study suggest that the HANDS app was successful in promoting social engagement through its mobile interventions because it does not overwork the individuals cognitive processing of verbal commands [16].

Mobile app technology has been utilized in vocational training for transition-age (18-24) youth with ASD. In a study of 15 young adults in a transition program, researchers examined the use of a mobile video modeling and prompting application, "VidCoach", in supporting the learning of interview skills for employment [17]. All except for one of the participants in the experimental group were 18 years of age and each participant was able to utilize the mobile app on their iOS devices or on an iPod touch. The VidCoach application provided pre-prompts to participants such as, "Hand the interviewer your resume", and post-prompts like "How long should you shake hands?" The goal of this mobile app technology is to help an individual's practice of modeled interview behaviors and reinforce their learned self-modeled behavior. Users of VidCoach mobile technology are able to watch modeling videos, record their own practice of the skills being modeled in the video, and review a video of themselves practicing the skill. As a result of utilizing this mobile app, all participants reported feeling more relaxed and confident in their interview skills and were more highly assessed by employers [17].

Researchers performed t-tests to evaluate whether job interview performance differed between the control and experimental groups at pre/post-interview and within the first/last two sessions. It was found that participants who used VidCoach fidgeted significantly less often, $t(316)=2.31, p=0.022$, presented ideas logically and succinctly significantly more often, $t(332)=-$ 2.64, $p=0.009$, and showed significant improvement in hygiene and hair care, $t(13)=-2.69, p=0.02$ [17]. However, participants in the control group were more likely to use correct grammar and vocabulary than participants who had used VidCoach, $t(267)=$ $-3.44, p<0.001$ [17]. Researchers were unable to explain the underlying mechanism for this difference, though it was noted that the scripts used in VidCoach were casual, compared to the more formal scripts used in schools. Their results indicated that use of the app directly before an interview can positively impact individuals in increasing their abilities to present their ideas logically and succinctly, improving interview hygiene, and decreasing fidgeting [17]. Results also suggested the use of this mobile app technology was enjoyable and considered useful by students in transition programs.

\section{Vocational interventions}

Social integration in the workplace is an integral aspect that possesses the capacity to improve the overall well-being of individuals with ASD. Often, individuals with ASD who are in the workplace, are able to undergo workplace training programs, such as Aspirations program, TEACCH supported employment program, or Project Search, that are focused on enhancing the social communication as well as emotional well-being of these individuals [18-20]. Likewise, these training programs foster psycho education of key workplace principles that foster social integration at work. It is imperative to acknowledge that these programs aim at enabling the workers with ASD to develop better workplace social behaviors, especially when relating to others. Such programs enhance their communication abilities in the workplace thereby making it possible for these individuals to effectively relate with others and foster effective integration into the organizational workforce. 
It has been found that vocational training programs also teach the ASD individuals to control their emotions, which is critical in enhancing their ability to become fully integrated into the social groups at places of work. Hence, through social integration that is achieved via such workplace training programs, the overall wellbeing of individuals with ASD is enhanced thus enabling them to cope well and have a healthy adjustment. The absence of such workplace training programs can result in individuals becoming socially isolated and create social isolation in workplaces. The resulting effect is poor emotional development and inability to communicate with other workers that translate to impaired wellbeing of these individuals.

Furthermore, social integration for individuals diagnosed with ASD can be improved via aspiration programs that aim at improving the overall well-being of individuals with ASD [21]. These aspiration programs may cover topics such as the development of effective social communication skills, building strong relationships, individual independence, as well as overcoming social challenges that may arise from being unemployed. Through these aspiration programs, adults diagnosed with ASD can come together and become socially integrated as they learn to develop new skills in the social, psychological as well as intellectual domains. Thus, the overall well-being of such individuals is enhanced.

In a study of social and vocational skills training, a discussionbased Aspirations program consisted of eight one-hour weekly small group meetings that aimed to improve the social and vocational skills [21]. A group of 36 participants completed questionnaires and measures such as Beck's Depression Index (BDI), and an Index of Peer Relations (IPR) to measure attitudes and feelings participants had towards their peers [21]. Results from their study found that participants ended with significantly reduced anxiety after their participation in the Aspirations programs as results from the State-Trait Anxiety Inventory (STAI) proved. Lower BDI scores were seen following participation in this program with 34 or $77 \%$ of participants reporting reduced depression. Improvement in participant's attitudes and feelings toward peers was also seen based on improvements on the Peer Relations Index (PRI) measure post-program [21]. It is clear that Aspiration programs influence the well-being of individuals with ASD by reducing anxiety and depressed symptomology through the program, all while increasing social engagement through group-based discussions.

Taylor et al. [22] studied the relationship between behavioral functioning symptoms and vocational activities. Vocational activities are a form of social engagement in which there is an opportunity for social inclusion rather than isolation. In a study of 153 individuals with ASD, more vocational independence was associated with less maladaptive behaviors and improved behavioral development. Vocational activities provide an opportunity for consistent social interaction that is a key part of creating a feeling of self-importance and meaning that enhances the well-being of these individuals. The findings of Taylor et al. [22] support that isolation and limited vocational involvement negatively impacts adult development and well- being in individuals with ASD just as it would among individuals without ASD who are unemployed.

\section{Multimedia social skills intervention}

Radley et al. examined the social skills needed to promote social engagement in young elementary school-age individuals with ASD. They implemented an intervention called, Superheroes Social Skills, in which videos of animated superhero characters modeled Social engagement was defined as direct social interaction with a peer in a class setting. They found that the evidence-based Superheroes Social Skills intervention increased social engagement in these children and as a result, they were better able to make friends and develop important cognitive and language skills; both have been described as significant factors in the well-being of individuals with ASD. Though these improvements were found, the sample sizes of only two young children in a school-based setting, and lack of application across developmental ages serve as limitations.

\section{Individualized interests in social engagement sports and games}

Research asserts that social integration among adults with autism spectrum disorder can be enhanced through combining social intervention tactics with adult physical activities [23]. As such, this approach fosters the development of both social skills as well as physical health. There are studies that maintain that the ability to develop these socialization skills possess the capacity to prevent or correct negative outcomes such as depression, individual anxiety as well as physical health challenges [23]. For instance, adults with ASD can become actively involved in physical activities such as games or sports [24]. This gives them the capacity to interact and develop connections with friends thereby fostering a sense of belonging [23]. The resultant effect is enhanced overall well-being after active involvement in such activities targeting both physical and social skills [24].

Nevertheless, when adults with ASD are not socially integrated and lack the opportunity to participate in such activities, both their physical and social well-being is impaired [23]. Hence, primary deficiencies are maintained while secondary deficiencies develop thereby impairing the individual's overall well-being [23]. A study on a 14-week Social Skills and Sports (S3) Program that combines classroom instruction with soccer activities examined its effect on improving social skills in four individuals with ASD aged 1424 [24]. The social skills targeted were contributing relevant information to a conversation, taking turns in conversation, and making appropriate eye contact. All of the participants showed improvements in their ability to demonstrate at least one of the targeted skills, generalize the skill(s) to other settings, and maintain the skill(s) five weeks following the intervention [24]. Participants had also shown the establishment of social skills that were not targeted in the S3 program [24].

\section{Music-based intervention}

Social integration entails the interaction of individuals in a manner that brings about individual fulfillment following the 
ability to take an active part in the group activities [25]. LaGasse [25] identifies that activities of social engagement, such as music therapy, possess the capacity to enhance the well-being of adults diagnosed with ASD. For example, individuals may be actively involved in peer-mediated activities such as music-based therapy in which they have an opportunity to be fully involved through turn-taking. Through turn-taking, the individual is able to take a leading role in communication with their peers, and thus are able to develop effective social skills. In a study of individuals with ASD, there were significant improvements in the initiation of communication, response to the communication or social withdrawal/behaviors, following weekly 50-minute sessions of group music therapy [25]. To evaluate these improvements, the Social Responsiveness Scale (SRS), Autism Treatment Evaluation Checklist (ATEC), and video analysis were used [25].

The use of music therapy in promoting social engagement has also been studied within a family-centered approach [26]. Researchers look for improvement in social engagement abilities in 23 children with a severe autism spectrum disorder, 12 of which were also in early intervention programs. Measurements used in determining improvements in social engagement were Social Responsiveness Scale Preschool Version (SRS- PS), ParentChild Relationship Inventory (PCRI), and a few subscales of the Vineland Social- Emotional Early Childhood Scales (VSEEC): interpersonal relationships subscale; and play and leisure time subscale. Social engagement was also measured using parent interviews, parent- report assessments, and clinician observation [26]. Family-centered music therapy (FCMT) was shown to improve social engagement for the participants in their home, in the community, and in parent-child interactions [26]. However, FCMT did not improve language skills and social responsiveness, other areas of socialization that may require further language and communicative skills-focused interventions [26].

\section{Social media use}

Several researchers have examined various interventions for developing social skills and encouraging appropriate social interactions in people with developmental disabilities. Appropriate interactions are those such as responding to questions such as "how are you?" response to compliments, greeting others upon meeting them, initiating a conversation where plausible, and carrying out a conversion on topic. However, there are few studies that examine how socialization might be facilitated through emerging technology. For this review, socialization has been defined as the process of acquiring a personal identity and learning the norms, values, behaviors, and social skills appropriate to an individual's social role [27].

Today, there are billions of people who communicate with others across the globe solely through social media, connecting people who may not have been connected before. Individuals with developmental disabilities have been able to utilize social media and connect with family and friends in a way that promotes healthy socialization and integrates them with society in a way where they are able to feel included [28]. Thus, social media use should be considered as a tool to alleviate social anxiety in individuals with developmental disabilities who are able to learn and utilize social media, though caution and protective considerations must also be carefully considered [28]. There are studies that suggest social media use could be beneficial in several areas of social engagement that could be applied to individuals with developmental disabilities. Research has found that among 108 adults with ASD ranging in age from 18-62 years, who used social media, most used social media to establish social connections [29]. Through the use of self-report measures, it was found that those who used social media for social engagement had closer friendships and more close friends. Decreased loneliness was a factor not directly resulting from social media use itself; however, it is suggested that the increase in friends that accompanied social media use, accounts for the decreased loneliness [30].

A study of 106 participants (64 male) diagnosed with Asperger's Disorder (56.6 percent), ASD (25.4 percent), and Autism (17.0 percent), examined social media use and happiness, the average age of participants being 28 years old [29]. It was found that adults with ASD who used SM reported greater subjective happiness than adults with ASD who did not use SM, and those who used Facebook more were happier than those who used Twitter more [29]. In this study, happiness is defined as the affective component of well-being, which was measured in the use of the Subjective Happiness Scale (SHS), a four-item 7- point scale that measures global subjective happiness, in which higher mean scores indicate greater happiness [29]. Research suggests that individuals who use SM experience greater social support with improved well-being that may relate to happiness [31].

Facebook and Twitter are noted to vary in the kind of social interactions users experience and thus may explain why Facebook users were happier than Twitter users [29]. Researchers provided the example of how Facebook is reciprocal in social interaction with the requirement of having to accept friend requests, whereas on Twitter, making a connection is unidirectional, where Tweeters follow others and there are no automatic reciprocated connections. Findings from this study suggest the well-being of individuals may be readily enhanced, given the use of social media sites like Facebook, in moderation. Participants used their favorite social media (SM) site an average of 2.3 hours a day, with 4.5 average hours of total SM use. No significant differences among self-reported diagnosis and choice of SM sites were found, v2 (14)=10.64, p=0.78 [29]. Prior research has shown that SM users, specifically Facebook users, enjoy increased social connections, improved trust, and increased well-being [32].

In another study, researchers examined social media use among 44 adolescents with ASD in comparison to a control group of 56 individuals without ASD [28]. In their study, social media use was significantly related to high-quality friendships, and the relationship between social media use and friendship quality was moderated by their anxiety levels. Analysts used the Social Media Experience Scale (SMES), which was developed specifically for 
this study, to assess individuals' experiences with social media. In considering well-being, the presence of quality friendships increases the likelihood that individuals can live socially engaged lives where they feel included among peers. It is important to note that perhaps increased exposure to social media could aid in decreasing any anxiety associated with its use in an exposure therapy like fashion. It is equally important to take into consideration any endangerment or harm that could arise from social media use.

\section{Virtual reality/online gaming}

Screen-based media and online gaming use have been investigated among 85 individuals with ASD between 14 and 60 years of age, and a control group of 71 participants without ASD [33]. Results indicated that those who played online games had more friends than those who did not engage in online gaming. Screen-based media use provides the opportunity for social engagement in such a way that omits the face to face difficulties with communication seen among individuals with ASD [33]. The investigation found that screen-based media and online gaming use enhanced social interaction, encouraged the building of social relationships, and leads to decreased loneliness reported among individuals.

Another study looked at how an independently controlled virtual 3D simulation intervention can improve social engagement through increased social understanding and skills in three participants aged 10-12 [34]. Their study acknowledged the benefit to individuals with ASD and their families' quality of life that technological advances such as virtual reality bestow. Also, this study referred to a number of researchers that have noted that children with ASDs have short attention spans that make it difficult for them to focus on continuous learning [34]. In this reference, the researchers of this study asserted that using virtual reality mitigates this limitation and decreases any distractions that may come from regular environments [34].

A 3D virtual bus stop and classroom environment were selected, as suggested by ASD experts, teachers, and parents of children with ASDs, and are noted to provide frequent opportunities to practice social interactions [34]. The intervention involved 3D social modeling, promotion, and awards of reinforcement, along with Twenty-four problem-based social questions included in the system. A few examples of the kinds of questions read aloud by the system are as follows: "Would you like to choose a seat on the bus?", "Can I talk loudly on the bus?", and "Why can't I do this?" [34]. Questions assessed non- verbal communication, social interactions, and social cognition. Cheng and colleagues (2015) developed two scales, social events card (SEC) and social behaviors scale (SBS), to measure each participant's social behaviors following the intervention. The target behaviors of social interaction, non-verbal communication, and social cognition were assessed with these measurements. Both measurement tools were assessed for validity and suitability by ASD experts and professionals. The results of this study indicated that social understanding and social skills improved in participants following the implementation of the system intervention [34].

\section{Discussion}

Autism Spectrum Disorder is a significant condition that impairs the ability of the adults to function typically in their social settings. As such, individuals are at risk for thwarted social lives, which are linked to poor social outcomes such as social isolation. Research shows that integration through interventions for social engagement can improve the well-being of individuals with autism. Social engagement improves their well-being in several ways such as promoting the formation of positive social relationships, empowering individuals with skill sets, reducing loneliness, and alleviating behaviors that may get in the way of their ability to engage socially with others. On the contrary, failure to develop social engagement translates to social isolation that would, in turn, harm the overall well-being of such individuals.

This reviewstressestheimportance of using availableopportunities for social engagement such as social skills interventions, training programs, and involvement in individualized interests that promote positive social interaction. It is increasingly becoming more common for social interventions for individuals with autism to use emerging technologies in teaching and encouraging social engagement. Research supports that there are significant gains to arise from involvement in activities and/or interventions that promote social engagement. These gains contribute to improving or enhancing the well-being of individuals with autism where not possible in individuals who are not supported through these approaches and are socially isolated. Individual differences in responses to interventions may very well exist, so it is imperative that interventions for social engagement are continuously explored to meet the individual needs of any person with an autism spectrum disorder.

\section{Conclusion and Future Prospects}

Future considerations should examine the implications of these forms of social engagement in improving well-being of individuals with comorbid mental illnesses such as anxiety and depression, which are most commonly seen across individuals with ASD diagnosis. Also, measurement of well-being should be explored with consideration of mediating factors such as culture, family environment, and availability of community resources. More research is needed to examine the role of ethnic/ cultural differences in the expression of and influence of social engagement on the well-being of individuals with ASD. These factors may help to further explain the mechanisms by which certain forms of social engagement are more or less effective based on individual characteristics such as differences in severity of ASD diagnosis and individual preferences. Furthermore, differing levels of ability and functioning should be more closely considered to determine if the effects of these forms of social engagement are transcribable across varying individual strengths and weaknesses seen across the autism spectrum. Research remains limited in the area of understanding mechanisms of influence of interventions on specific comorbid mental illnesses, relative to the bidirectionality of the influence of challenges faced by individuals with ASD and comorbid anxious or depressive symptoms on social functioning and vice versa. 


\section{References}

1 Avison WR, McLeod JD, Pescosolido BA (2007) Mental health, social mirror Springer.

2 Sims J (2013) Public health for an ageing society edited by T.R. prohaska, L.A. anderson and R.H. binstock the johns hopkins university press, baltimore, maryland, USA. Australas J Ageing 32: 194-195.

3 Orsmond GI, Shattuck PT, Cooper BP, Sterzing PR, Anderson KA (2013) Social participation among young adults with an autism spectrum disorder. J Autism \& Dev Dis 43: 2710-2719.

4 Spain D, Sarah HB (2015) Group social skills interventions for adults with high- functioning autism spectrum disorders: A systematic review. Autism 19: 874-886.

5 Hotton M, Coles S (2016) The effectiveness of social skills training groups for individuals with autism spectrum disorder. Review Autism \& Dev Dis 3: 68-81.

6 Karst JS, Van AV, Carson AM, Stevens S, Schohl K, et al. (2015) Parent and family outcomes of PEERS: A social skills intervention for adolescents with autism spectrum disorder. J Autism \& Dev Dis 45: 752-765.

7 White SW, Roberson-Nay R (2009) Anxiety, social deficits and loneliness in youth with autism spectrum disorders. J Autism \& Dev Dis 39: 1006-1013.

8 Attwood T (2006) The complete guide to Asperger's syndrome. Jessica Kingsley Publishers.

9 Sterling L, Dawson G, Estes A, Greenson J (2008) Characteristics associated with presence of depressive symptoms in adults with autism spectrum disorder. J Autism \& Dev Dis 38: 1011-1018.

10 Mazurek MO (2014) Loneliness, friendship and well-being in adults with autism spectrum disorders. Autism 18: 223-232.

11 Yoo H, Bahn G, Cho I, Kim E, Kim J, et al. (2014) A randomized controlled trial of the korean version of the PEERS ${ }^{\circledR}$ parent-assisted social skills training program for teens with ASD. Autism Res 7 : 145-161.

12 Sivberg B (2002) Family system and coping behaviors: A comparison between parents of children with autistic spectrum disorders and parents with non-autistic children. Autism 6: 397-409.

13 Lam S, Wong BPH, Leung D, Au-Yeung P (2010) How parents perceive and feel about participation in community activities: The comparison between parents of preschoolers with and without autism spectrum disorders. Autism 14: 359-377.

14 Kamps D, Royer J, Dugan E, Kravits T, Gonzalez-Lopez A (2002) Peer training to facilitate social interaction for elementary students with autism and their peers. Exceptional Child 68: 173-187.

15 Bambara LM, Cole CL, Kunsch C, Tsai S, Ayad E (2016) A peermediated intervention to improve the conversational skills of high school students with autism spectrum disorder.

16 Mintz J (2013) Additional key factors mediating the use of a mobile technology tool designed to develop social and life skills in children with autism spectrum disorders: Evaluation of the 2nd HANDS prototype.

17 Hayes GR, Custodio VE, Haimson OL, Nguyen K, Ringland KE, et al.
(2015) Mobile video modeling for employment interviews for individuals with autism. Journal of Vocational Rehabilitation 43: 275-287.

18 Hillier A, Campbell H, Mastriani K, Izzo MV, Kool-Tucker AK, et al. (2007) Two-year evaluation of a vocational support program for adults on the autism spectrum. Career Dev Transition Except Individ 30: 35-47.

19 Keel JH, Mesibov GB, Woods AV (1997) TEACCH-supported employment program. J Autism \& Dev Dis 27(1), 3-9.

20 Rutkowski S, Daston M, Van KD, Riehle E (2006) Project SEARCH: A demand-side model of high school transition. J Vocat Rehabil 25: 85-96.

21 Hillier AJ, Fish T, Siegel JH, Beversdorf DQ (2011) Social and vocational skills training reduces self-reported anxiety and depression among young adults on the autism spectrum. J Dev \& Phys Disabil 23: 267-276.

22 Taylor JL, Smith LE, Mailick MR (2014) Engagement in vocational activities promotes behavioral development for adults with autism spectrum disorders. J Autism \& Dev Dis 44: 1447-1460.

23 Kunzi K (2015) Improving social skills of adults with autism spectrum disorder through physical activity, sports, and games: A review of the literature. Adultspan J 14.

24 Alexander M, Dummer GM, Smeltzer A, Denton SJ (2011) Developing the social skills of young adult special Olympic athletes. Edu and Train in Autism \& Develop Disab 46: 297-310.

25 LaGasse $A B$ (2017) Social outcomes in children with autism spectrum disorder: A review of music therapy outcomes. Patient Related Outcome Measures 8: 23.

26 Thompson GA, McFerran KS, Gold C (2014) Family-centred music therapy to promote social engagement in young children with severe autism spectrum disorder: A randomized controlled study. Child Care, Health \& Dev 40: 840-852.

27 Schneewind KA (2015) In Wright J. D, Socialization and education: Theoretical perspectives.

28 Van GI, Marin CE, Ortiz M, Rolison M, Qayyum Z, et al. (2017) Social media use, friendship quality and the moderating role of anxiety in adolescents with autism spectrum disorder. J Autism \& Dev Dis 47: 2805-2813.

29 Ward DM, Dill-Shackleford KE, Mazurek MO (2018) Social media use and happiness in adults with autism spectrum disorder. Cyberpsychol Behav Soc Netw 21: 205-209.

30 Mazurek MO (2013) Social media use among adults with autism spectrum disorders.

31 Carter S, Yeo AC (2018) Internet-enabled collective intelligence as a precursor and predictor of consumer behaviour. Economics, Management and Financial Markets 13: 11.

32 Valenzuela S, Park N, Kee KF (2009) Is there social capital in a social network site? Facebook use and college students' life satisfaction, trust and participation J Comput-Mediat Commun 14: 875-901.

33 Carvalho SA, Fernandes GM, Furtado RI, Alves BC (2015) Intervention in autism: Social engagement implemented by caregivers. Paideía 25: 67.

34 Cheng Y, Huang C, Yang C (2015) Using a 3D immersive virtual environment system to enhance social understanding and social skills for children with autism spectrum disorders. Focus on Autism and Other Developmental Disabilities 30: 222-236. 